\title{
ENFERMEDAD ARTERIAL PERIFÉRICA EN PACIENTES DE UN SERVICIO DE CLÍNICA MÉDICA
} EN PARAGUAY

\section{PERIPHERAL ARTERIAL DISEASE IN PATIENTS OF AN INTERNAL MEDICINE DEPARTMENT} IN PARAGUAY

Raúl Emilio REAL ${ }^{1}$, Nicolás Emilio REAL-APARICIO ${ }^{2}$, Esteban Andrés SANTANDER ${ }^{2}$, Luis GIMÉNEZ ${ }^{2}$, Hubert LEGUIZAMÓN ${ }^{2}$, María Eugenia ACOSTA ${ }^{3}$.

\author{
${ }^{1}$ Médico Internista, Departamento de Medicina Interna, Hospital Nacional, Itauguá - Paraguay. \\ ${ }^{2}$ Estudiantes de Medicina, Facultad de Ciencias Médicas, Universidad del Pacífico, Asunción - Para- \\ guay. \\ ${ }^{3}$ Bioquímica, Facultad de Ciencias Médicas, Universidad del Pacífico, Asunción - Paraguay.
}

Cómo citar este artículo: Real R, Real-Aparicio N, Santander EA, Giménez N, Leguizamón H, Acosta ME. Enfermedad arterial periférica en pacientes de un servicio de clínica médica en Paraguay. Medicina Clínica y Social. 2017;1(3):201-208.

\section{RESUMEN}

Introducción: la enfermedad arterial periférica es una afección frecuente en adultos mayores. Se acrecienta con la presencia de comorbilidades. Puede ser detectada por la medición de la presión arterial en los cuatro miembros y el cálculo del índice tobillo-brazo. Objetivos: determinar la frecuencia de enfermedad arterial periférica detectada por el índice tobillo-brazo, los síntomas de claudicación intermitente de miembros y la presencia de comorbilidades. Metodología: estudio observacional descriptivo prospectivo de corte transversal realizado en 62 pacientes mayores de 40 años internados en el Servicio de Clínica Médica del Hospital Nacional (Itauguá, Paraguay) en el 2016. Se determinó la presión arterial en los cuatro miembros con un tensiómetro digital y se registró la presencia de comorbilidades. El índice tobillo-brazo derecho se calculó por el cociente de la presión arterial sistólica del tobillo derecho dividido la presión arterial sistólica más elevada de cualquier brazo y de forma similar para el índice tobillo-brazo izquierdo. Se consideró índice tobillo-brazo compatible con enfermedad arterial periférica todo valor $\leq 0,9$. Resultados: en la muestra predominó el sexo masculino (52\%) y la edad media fue $54 \pm 9$ años. Las comorbilidades más frecuentes fueron hipertensión arterial (48\%), tabaquismo (32\%) y diabetes mellitus (26\%). Se detectó enfermedad arterial periférica en 9,6\%. La mayoría de los afectados se encontraban en el grado I de la clasificación de Fontaine (asintomáticos). Discusión: la frecuencia de enfermedad arterial periférica fue $9,6 \%$. Sólo un paciente era sintomático. Las comorbilidades más frecuentes fueron la hipertensión arterial y el tabaquismo. La determinación de la presión arterial en los cuatro miembros permite detectar una enfermedad asintomática, potencialmente grave.

Palabras clave: Enfermedad arterial periférica; Índice tobillo-brazo; Hipertensión arterial; Diabetes mellitus; Tabaquismo.

\section{ABSTRACT}

Introduction: Peripheral arterial disease is a common condition in older adults. It increases with the presence of comorbidities. It can be detected by measuring blood pressure in the four limbs and calculating the ankle-brachial index. Objectives: To determine the frequency of peripheral arterial disease detected by ankle-brachial index, the symptoms of intermittent limb claudication and the presence of comorbidities. Methodology: Cross - sectional, prospective, observational study of 62 patients over 40 years of age admitted to the Clinical Service of the National Hospital (Itauguá, Paraguay) in 2016. 
Blood pressure was determined in the four limbs with a digital blood pressure monitor and the presence of comorbidities was recorded. The right ankle-brachial index was calculated with the systolic blood pressure of the right ankle divided by the highest systolic blood pressure of any arm and similarly for the left ankle-brachial index. A value of $\leq 0.9$ was considered compatible with peripheral arterial disease. Results: In the sample predominated the male sex (52\%) and the mean age was $54 \pm 9$ years. The most frequent comorbidities were arterial hypertension $(48 \%)$, smoking (32\%) and diabetes mellitus (26\%). Peripheral arterial disease was detected in $9.6 \%$. The majority of those affected were in grade I of the Fontaine classification (asymptomatic). The value of this method is detecting a potentially serious asymptomatic disease. Discussion: the frequency of peripheral arterial disease was $9.6 \%$. Only one patient was symptomatic. The most frequent comorbidities were arterial hypertension and smoking.

Keywords: Peripheral arterial disease; Ankle-brachial index; Arterial hypertension; Diabetes mellitus; Smoking.

\section{INTRODUCCIÓN}

La enfermedad arterial periférica (EAP) es una afección de los grandes vasos y arterias de mediano y pequeño calibre debida a la acumulación de placas ateromatosas originando un estrechamiento en la luz de los mismos con trastornos en la circulación distal. Es una manifestación más de la aterosclerosis sistémica y, por tanto, depende de los factores de riesgo cardiovascular (1,2).

La prevalencia de la EAP en la atención primaria es 5-30\%. Esta frecuencia varía con la edad: en el grupo de 60-65 años es 35\% pero entre los $70-75$ años la prevalencia se incrementa hasta alcanzar 70\% $(1,3)$. Los factores de riesgo implicados en la aparición de la EAP son los de riesgo cardiovascular: hipertensión arterial, diabetes mellitus, tabaquismo, dislipidemia, hiperhomocisteinemia, sexo masculino, obesidad y vida sedentaria (4-6).

Únicamente el $10 \%$ de los individuos con EAP presenta la sintomatología clásica de claudicación intermitente de miembros que se presenta con los esfuerzos físicos (7-9). La clasificación de Fontaine categoriza a los síntomas y signos según la distancia que debe recorrer un paciente para que aparezca el dolor.

Existen diversos métodos diagnósticos para la EAP. La angiografía tiene una alta especificidad y sensibilidad por lo que se la considera el gold standard, pero es invasiva y costosa $(1,10)$. La ecografía Doppler es una técnica menos costosa y más segura, pero es operador dependiente y requiere equipos de alta tecnología. La palpación de los pulsos periféricos es una técnica semiológica fácil, pero puede generar falsos negativos. El índice tobillo-brazo (ITB) consiste en determinar la presión arterial en los cuatro miembros y calcular la relación entre miembros inferiores y superiores. Es una técnica con $95 \%$ de sensibilidad y una especificidad próxima al $100 \%$. Además, es un procedimiento barato, preciso, rápido y reproducible. Esta gran precisión diagnóstica lo convierte en el método diagnóstico de elección a la cabecera del paciente, por lo que se utilizó en esta investigación (11-17). Sólo se precisa un tensiómetro manual o digital. Este último ha demostrado suficiente capacidad diagnóstica en diversos estudios (1822).

Existe evidencia para relacionar la EAP con una mayor tasa de infarto de miocardio, ictus y muertes de origen vascular (23). Los pacientes asintomáticos con EAP tienen un elevado riesgo de enfermedades cardiacas, por lo que la búsqueda sistemática de EAP en pacientes con factores de riesgo cardiovascular adquiere una relevancia clínica importante. Las complicaciones 
locales son los trastornos tróficos (caída de vellos, anhidrosis, piel delgada y con fisuras), ulceraciones y mala cicatrización de heridas. El paciente con EAP en etapas avanzadas de su patología se encuentra en alto riesgo de amputación por necrosis de sus miembros (24-29).

El tratamiento esencial consiste en actuar sobre los factores de riesgo modificables, mejorando la hipertensión arterial, optimizando la glicemia en los diabéticos, reduciendo la dislipidemia, bajando el peso a los obesos y suprimiendo el tabaquismo. Existen medicamentos que han demostrado efectividad: antiagregantes plaquetarios (ácido acetil salicílico, clopidogrel), hemoreológicos (pentoxifilina) e inhibidores de la fosfo-5-diesterasa (cilostazol) (25).

La EAP debe ser una afección frecuente en la población paraguaya adulta dada la alta frecuencia de factores de riesgo cardiovascular en nuestro país: obesidad (30\%), hipertensión arterial $(30,5 \%)$, diabetes mellitus $(6,5 \%)$, sedentarismo $(40 \%)(30,31)$. La EAP se caracteriza por ser asintomática en alta proporción (90\%) y porque sus complicaciones son graves e invalidantes $(1,25)$. La detección con la prueba ITB con tensiómetro digital es una técnica muy fiable. Por todo lo expuesto se planteó esta investigación con el propósito de diagnosticar sujetos asintomáticos y que puedan beneficiarse de un tratamiento oportuno (32).

\section{METOdOLOGÍA}

Se realizó un estudio observacional, descriptivo, de corte transversal. Se encontraron 280 pacientes internados en cuatro visitas al Servicio de Clínica Médica, de los cuales 62 reunían los criterios de inclusión y aceptaron participar del estudio. Los excluidos fueron debidos a tener menos de 40 años, edema de miembros o amputados o por falta de colaboración; un caso fue excluido por tener miembro amputado por EAP.

Se utilizó el tensiómetro digital marca Digit Smart PIC Solution ${ }^{\circledR}$ (Italy) para la aplicación de la medición del ITB. Este índice es el resultado de dividir la presión arterial sistólica (PAS) de cada tobillo (se escogerá el valor más alto entre la arteria pedia y la tibial posterior) entre el valor de la PAS más alto de cualquiera de las arterias braquiales de ambos brazos (1). Así se obtienen dos valores de ITB, uno para cada miembro inferior. Un índice $<0,9$ es diagnóstico de EAP, pero un valor menor a 0,4 es diagnóstico certero de EAP severa (1).

Los factores de riesgo cardiovascular fueron extraídos de los expedientes médicos. Las variables fueron registradas en una planilla electrónica Excel $^{\mathrm{TM}}$ y sometidas a estadística descriptiva con el programa informático Epi Info 7(C. Las variables cualitativas se expresaron en frecuencias y porcentajes y las cuantitativas en medias y desvíos estándar o percentiles.

Se respetaron los Principios de la Bioética. Todos los sujetos que cumplían con los criterios de inclusión tuvieron la posibilidad de ser estudiados. No se hizo discriminación por edad, sexo o religión. Los pacientes decidieron voluntariamente participar del estudio. A todos se les leyó el consentimiento informado, se les aclaró las dudas y firmaron su acuerdo a ser evaluados. Los resultados del estudio fueron entregados al médico tratante y a los pacientes, quienes podrán beneficiarse de tratamientos según cada caso. Los gastos de esta investigación corrieron por cuenta de los autores. El protocolo de investigación fue aprobado por el Comité de Ética de la Universidad del Pacífico y contó con la anuencia de las autoridades del Hospital Nacional. 


\section{RESULTADOS}

La edad media de los sujetos fue $54 \pm 9$ años (rango 40-74 años). Hubo un ligero predominio del sexo masculino: 32 casos (52\%). La comorbilidad más frecuente fue la hipertensión arterial (47\%) (Tabla 1).

\begin{tabular}{|c|c|c|}
\hline \multicolumn{2}{|c|}{ TABLA 1. FRECUENCIA DE COMORBILIDADES ( $\mathbf{n = 6 2 )}$} \\
\hline Comorbilidades & Frecuencia & Porcentaje \\
\hline Hipertensión arterial & 30 & 48 \\
Tabaquismo & 20 & 32 \\
Diabetes mellitus & 16 & 26 \\
Dislipidemia & 16 & 26 \\
Obesidad & 8 & 13 \\
\hline
\end{tabular}

En 37 sujetos (60\%) la PAS sobrepasaba $130 \mathrm{~mm}$ Hg. La PAS más elevada en cualquier miembro superior tuvo una mediana de $137 \mathrm{~mm} \mathrm{Hg}$ con un rango intercuartílico de $124-191 \mathrm{~mm} \mathrm{Hg}$.

Calculando el ITB derecho se encontró $2 / 62$ (3,2\%) con EAP y 4/62 para el ITB izquierdo (6,4\%). Ninguno de ellos presentó sintomatología de claudicación de miembros, excepto por un varón de 74 años, portador de hipertensión arterial y tabaquismo, que presentaba ITB izquierdo 0,7 y derecho 0,6 con sintomatología de claudicación de miembros compatible con la escala lla del cuestionario de Fontaine (Figura 1).

Figura 1. Frecuencia de enfermedad arterial periférica según índice tobillo-brazo ( $n=62)$.

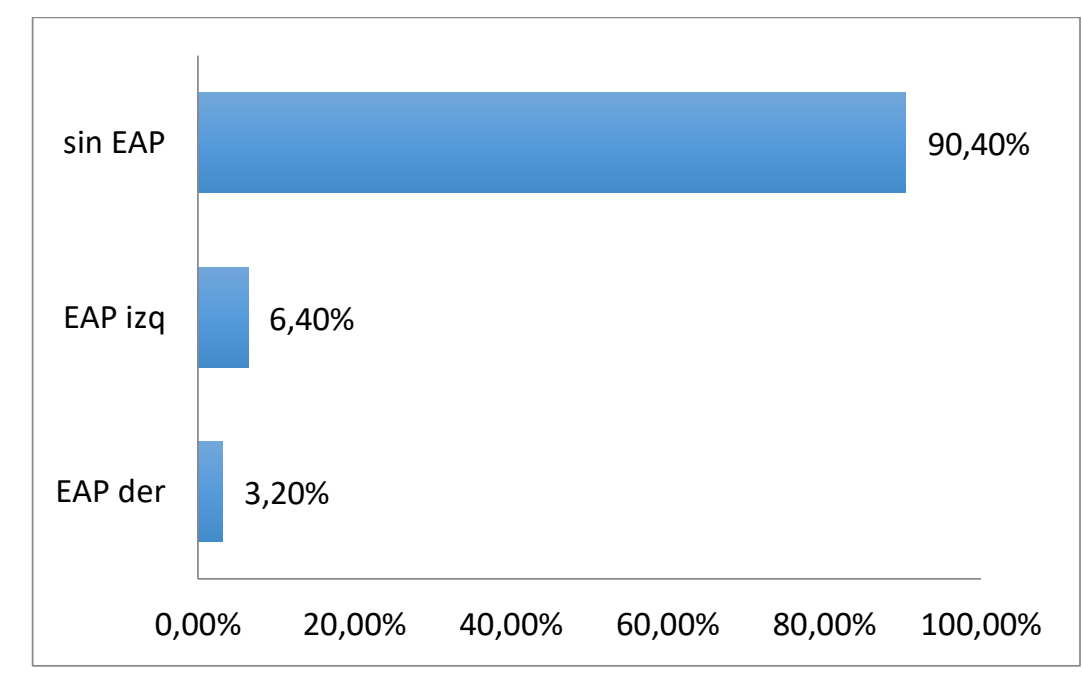

\section{DISCUSIÓN}

La frecuencia de ITB alterado detectado en esta investigación (3,2 y 6,4\%) coincide con la literatura consultada $(1,3)$. Llama la atención la elevada cantidad de pacientes con hipertensión arterial (30 pacientes, 48\%), dado que la prevalencia de hipertensión arterial en el Paraguay es de $20 \%$ en la población adulta $(30,31)$. Todos estos pacientes tenían controles seriados de presión arterial durante su internación por lo que se consideró a todos como hipertensos. Sin 
embargo, esta muestra no corresponde a una población representativa del país pues son pacientes internados mayores de 40 años.

La limitación de este estudio es que no se confirmó la presencia de EAP con otros métodos más sensibles, pero es sabido que la ecografía Doppler y la arteriografía no son métodos diagnósticos utilizados a la cabecera del paciente, sino de segunda línea. No obstante, la simple medición de la presión arterial en los cuatro miembros y el cálculo del ITB son de uso clínico pues es una técnica incruenta, sencilla, barata y reproducible (11-17). Sería ideal realizar esta misma investigación en una población no hospitalizada, aprovechando las campañas de detección de hipertensión arterial (31).

El hecho que un solo sujeto con EAP tuviera sintomatología de claudicación de miembros resultó inesperado pues todos los pacientes incluidos se movilizaban o al menos realizaban caminatas sin sentir molestias. Por esto sería interesante verificar la presencia de EAP por otros métodos (ecografía Doppler, arteriografía), dada la importancia de este diagnóstico por sus consecuencias a veces graves (5). No obstante, debido a su morbilidad y eventual mortalidad, se reserva la arteriografía solo en caso de planear una cirugía de revascularización arterial (bypass). Por ello, nunca deberá indicarse este método auxiliar de diagnóstico como un estudio rutinario tan solo para evaluar a un paciente en el cual se sospecha EAP.

Llamó la atención que de 37 pacientes que presentaban PAS $>130 \mathrm{~mm} \mathrm{Hg,} 15$ desconocían ser portadores de hipertensión arterial antes de su internación. Esto se debe a que la hipertensión arterial es asintomática y si no se realizan mediciones periódicas no podrá ser detectada oportunamente. De ahí viene la denominación de "la asesina silenciosa" (31).

En un estudio reciente se midió el ITB en sujetos jóvenes adultos de Canadá con edad media $24 \pm 2$ años y sin mayores comorbilidades y se encontró en varones un ITB izquierdo de 0,98 $\pm 0,12$ e ITB derecho $0,97 \pm 0,12$. En las mujeres la media fue $0,95 \pm 0,21$ y $0,94 \pm 0,21$, respectivamente (32). Sus autores concluyen que el umbral para el diagnóstico de EAP basada en la medición del ITB debe aumentar dado que estos valores resultaron bastante bajos y sugestivos de EAP, en sujetos sanos y sin factores de riesgo cardiovascular. Estos hallazgos deberían replicarse en sujetos jóvenes de nuestro país.

La limitación de este estudio es que no se confirmó la presencia de EAP con otros métodos más sensibles. No obstante, la simple medición de la presión arterial en los cuatro miembros y el cálculo del ITB son de uso clínico pues es una técnica incruenta, sencilla, barata y reproducible. Sería ideal realizar esta misma investigación en una población no hospitalizada, aprovechando las campañas de detección de hipertensión arterial.

En conclusión, la frecuencia de EAP fue 9,6\%. Sólo un paciente presentó la sintomatología de la claudicación intermitente. Las comorbilidades más frecuentes fueron la hipertensión arterial (48\%) y el tabaquismo (32\%). Se destaca el valor de este método en detectar una enfermedad asintomática, potencialmente grave. Finalmente, el ITB es un método práctico, sencillo y barato, teniendo la salvedad que, en sujetos diabéticos de larga data con tratamiento irregular, el valor del ITB es relativo, debido a los resultados engañosos obtenidos de la medición de la presión arterial ocasionados por la intensa esclerosis de las arterias como consecuencia de la hiperglicemia crónica. 


\section{CONFLICTOS DE INTERÉS Y FUENTE DE FINANCIACIÓN}

Los autores declaran no poseer conflictos de interés. Fuente de financiación: ninguna.

\section{REFERENCIAS BIBLIOGRÁFICAS}

1. Serrano Hernando FJ, Martín Conejero A. Peripheral artery disease: pathophysiology, diagnosis and treatment. Rev Esp Cardiol. 2007;60(9):969-982. URL.

2. Huelmos A, Jiménez J, Guijarro C, Belinchón JC, Puras E, Sánchez C, et al. Underrecognized peripheral arterial disease in patients with acute coronary syndrome: prevalence of traditional and emergent cardiovascular risk factors. Rev Esp Cardiol. 2005;58(12):1403-1410. https://dx.doi.org/10.1016/S1885-5857(06)60747-0

3. Chen YW, Wang YY, Zhao D, Yu CG, Xin Z, Cao X, et al. High prevalence of lower extremity peripheral artery disease in type 2 diabetes patients with proliferative diabetic retinopathy. PLoS One. 2015;10(3):e0122022. https://dx.doi.org/10.1371\%2Fjournal.pone.0122022

4. Cho SW, Kim BG, Kim DH, Kim BO, Byun YS, Rhee KJ, et al. Prediction of coronary artery disease in patients with lower extremity peripheral artery disease. Int Heart J. 2015;56(2):209-212. https://doi.org/10.1536/ihj.14-284

5. Aiman U, Haseen MA, Beg MH, Khan RA, Siddiqui FA, Alam I. Profile of atherosclerotic risk factors and management in patients of peripheral arterial disease at a tertiary care teaching hospital of north India. Indian J Pharm Sci. 2014;76(6):504-509. URL.

6. Valdivielso P, Ramírez-Bollero J, Pérez-López C. Peripheral arterial disease, type 2 diabetes and postprandial lipidaemia: Is there a link? World J Diabetes. 2014;5(5):577585. https://doi.org/10.4239/wjd.v5.i5.577

7. Hirsch AT, Criqui MH, Treat-Jacobson D, Regensteiner JG, Creager MA, Olin JW, et al. Peripheral arterial disease detection, awareness, and treatment in primary care. JAMA. 2001;286:1317-1324. https://doi.org/10.1001/jama.286.11.1317

8. Makdisse M, Nascimento Neto R, Chagas AC, Brasil D, Borges JL, Oliveira A, et al. Crosscultural adaptation and validation of the Brazilian Portuguese version of the Edinburgh Claudication Questionnaire. Arq Bras Cardiol.2007;88(5):501-506. https://doi.org/10.1590/S0066-782X2007000500001

9. Je HG, Kim BH, Cho KI, Jang JS, Park YH, Spertus J. Correlation between Patient-Reported Symptoms and Ankle-Brachial Index after Revascularization for Peripheral Arterial Disease. Int J Mol Sci. 2015;16(5):11355-11368. https://doi.org/10.3390\%2Fijms160511355

10. Werncke T, Ringe KI, von Falck C, Kruschewski M, Wacker F, Meyer BC. Diagnostic confidence of run-off CT-angiography as the primary diagnostic imaging modality in patients presenting with acute or chronic peripheral arterial disease. PLoS One. 2015; 10(3):e0119900. https://doi.org/10.1371/journal.pone.0119900

11. Maggi DL, Dal Piva de Quadros LR, de Oliveira Azzolin K, Goldmeier S. Ankle-brachial index: nurses strategy to cardiovascular disease risk factors identification. Rev Esc Enferm USP. 2014;48(2):223-227. https://doi.org/10.1590/S0080$\underline{623420140000200004}$

12. Kabul HK, Aydogdu A, Tasci I. Calculation of the ankle brachial index. Arq Bras Cardiol. 2012 Aug; 99(2):772-773. https://doi.org/10.1590/S0066-782X2012001100012 
13. Albuquerque PF, Albuquerque PH, Albuquerque GO, Servantes DM, Carvalho SM, Oliveira Filho JA. Ankle-brachial index and ventricular hypertrophy in arterial hypertension. Arq Bras Cardiol. 2012;98(1):84-86. https://doi.org/10.1590/S0066$782 \times 2012000100013$

14. Olalla J, Salas D, De La Torre J, Del Arco A, Prada JL, García Alegría J. Ankle-brachial index in the assessment of cardiovascular risk among HIV infected patients. Rev Med Chil. 2011;139(8):1039-1045. https://doi.org/10.4067/S0034-98872011000800009

15. Valdivielso P, Mancera-Romero J, Sánchez-Chaparro MA. Vascular risk, diabetes and the ankle-brachial index. Rev Esp Cardiol. 2011;64(8):729. https://doi.org/10.1016/j.rec.2011.03.029

16. Baena-Díez JM, Alzamora MT, Forés R, Pera G, Torán P, Sorribes M; ARTPER study. Ankle-brachial index improves the classification of cardiovascular risk: PERART/ARTPER Study. Rev Esp Cardiol. 2011;64(3):186-192. https://doi.org/10.1016/j.recesp.2010.10.024

17. Miguel JB, Strogoff de Matos JP, Ruzany F, Miguel CS, Miguel SJ, Naveiro LT, et al. Association of ankle-arm index with inflammation and mineral bone disorder in hemodialysis patients. Arq Bras Cardiol. 2011;96(5):405-409. https://doi.org/10.1590/S0066782X2011005000031

18. Vega J, Romaní S, Garcipérez FJ, Vicente L, Pacheco N, Zamorano J, et al. Peripheral arterial disease: efficacy of the oscillometric method. Rev Esp Cardiol. 2011;64(7):619621. https://doi.org/10.1016/j.recesp.2010.10.019

19. Aguilar-Shea AL. Ankle-arm index and cardiovascular prevention. Aten Primaria. 2011;43(3):162-163. https://doi.org/10.1016/j.aprim.2010.02.012

20. Méndez Abad M, Gorrín Vargas G, Francisco Rodríguez M, Ardevol González R. Anklearm index as a screening method in Primary Care for silent peripheral arterial disease. Aten Primaria. 2010;42(1):61-62. https://doi.org/10.1016/j.aprim.2009.02.015

21. Maldonado J, Pereira T, Resende M, Simões D, Carvalho M. Usefulness of the anklebrachial index in assessing vascular function in normal individuals. Rev Port Cardiol. 2008;27(4):465-476. URL.

22. Kawamura T. Assessing Ankle-Brachial Index (ABI) by using automated oscillometric devices. Arq Bras Cardiol. 2008;90(5):294-298. https://doi.org/10.1590/S0066782X2008000500003

23. Manzano L, García-Díaz J de D, Gómez-Cerezo J, Mateos J, del Valle FJ, Medina-Asensio $J$ et al. Clinical value of the ankle-brachial index in patients at risk of cardiovascular disease but without known atherothrombotic disease: VITAMIN study. Rev Esp Cardiol. 2006;59(7):662-670. https://doi.org/10.1157/13091367

24. Lahoz C, Mostaza JM. Ankle-brachial index: a useful tool for stratifying cardiovascular risk. Rev Esp Cardiol. 2006;59(7):647-649. https://doi.org/10.1157/13091364

25. Rooke TW, Hirsch AT, Misra S, Sidawy AN, Beckman JA, Findeiss LK, et al.2011 ACCF/AHA Focused Update of the Guideline for the Management of Patients With Peripheral Artery Disease (Updating the 2005 Guideline): A Report of the American College of Cardiology Foundation/American Heart Association Task Force on Practice Guidelines. J Am Coll Cardiol. 2011;58(19):2020-2045. https://doi.org/10.1016/i.jacc.2011.08.023

26. Sarmento C, Pereira T, Maldonado J, Conde J. Peripheral artery disease and kidney function in hypertensive patients. Arq Bras Cardiol. 2013;100(4):362-367. https://doi.org/10.5935/abc.20130052 
27. Tranche-Iparraguirre S, Marín-Iranzo R, Fernández-de Sanmamed R, Riesgo-García A, Hevia-Rodríguez E, García-Casas JB. Peripheral arterial disease and kidney failure: a frequent association. Nefrologia. 2012;32(3):313-320. https://doi.org/10.3265/Nefrologia.pre2011.Nov.11172

28. Swaminathan A, Vemulapalli S, Patel MR, Jones WS. Lower extremity amputation in peripheral artery disease: improving patient outcomes. Vasc Health Risk Manag. 2014; 10: 417-424. https://doi.org/10.2147/VHRM.S50588

29. Arévalo-Manso JJ, Juárez-Martín B, Fernández-Rodríguez $G$, Martínez-Sánchez $P$, ParriIla-Novo $P$, Pérez-Fernández $E$, et al. Diagnostic accuracy of an automatic blood pressure device for ankle brachial index determination in ischemic stroke patients. Rev Neurol. 2012;55(3):129-136. URL.

30. Jimenez JT, Palacios M, Cañete F, Barriocanal LA, Medina U, Figueredo R, et al. Prevalence of diabetes mellitus and associated cardiovascular risk factors in an adult urban population in Paraguay. Diabet Med. 1998;15(4):334-338. https://doi.org/10.1002/(SICI)1096-9136(199804)15:4\%3C334::AIDDIA575\%3E3.0.CO;2-Z

31. Ortellado Maidana J, Ramírez A, González G, Olmedo Filizzola G, Ayala de Doll M, Sano $M$, et al. Consenso Paraguayo de Hipertensión Arterial 2015. Rev Virtual Soc Parag Med Int. 2016;3(2):11-57. https://doi.org/10.18004/rvspmi/2312-3893/2016.03(02)11-057

32. Quong WL, Fung AT, Yu RY, Hsiang YN. Reassessing the normal toe-brachial index in young healthy adults. J Vasc Surg. 2016;63(3):652-656. https://doi.org/10.1016/i.jvs.2015.09.019 